\title{
Health preparedness and legacy planning at mass gatherings in the EMR: a WHO perspective
}

\author{
Nicolas Isla and Isabelle Nuttall ${ }^{2}$
}

As more countries commit to hosting large, international mass gatherings, health preparedness planning is requiring more research and collaborative efforts. A growing body of researchers and policy-makers view mass gatherings as important opportunities for positive legacy for the community and visitors. This special edition of the Eastern Mediterranean Health Journal is a good example of a respected scientific publication offering its pages to researchers to share their experiences studying small, medium and large events; religious, sporting and cultural events; one-off events and events that recur in the same location year after year. This work, and the work of others, will help future hosts to assess options, to adapt and build existing systems and to evaluate their effectiveness through the acquired experience and mounting evidence generated by past organizers.

The Eastern Mediterranean Region (EMR) is host to some of the world's largest mass gatherings. Each year the Kingdom of Saudi Arabia welcomes upwards of 3 million pilgrims from around the world; the Formula 1 motor racing championship is being held in two EMR countries in 2013 and Qatar is set to host the FIFA World Cup in 2022. There are a large number of other mass gatherings in the Region of all sizes and purposes, each with their own specific risks.

The World Health Organization (WHO) continues to build an interdisciplinary mass gathering programme that offers guidance, tools and expertise that public health authorities can use before planning an event. This mandate was provided by a decision at the $130^{\text {th }}$ Executive Board Meeting in January 2012 advising the Secretariat to, among other activities, "multisectoral guidance on planning, management, evaluation and monitoring of all types of mass gathering events with specific emphasis on sustainable preventive measures including health education and preparedness". In response, WHO has developed a three-point strategy for supporting countries with mass gathering health planning:

- Capacity development and support for Member States and host organizations - protecting public health at mass gatherings

- Establishment of governance, resources, tools and methodologies

- Shaping the discipline: leadership and communication.

WHO has worked with organizers of some of the largest events including the London Olympics and the 2012 UEFA European Football Championship in Poland and Ukraine, Hajj and the World Youth Day. In addition, over the past year, a network of Collaborating Centres on mass gatherings has been established to act as regional hubs for best practice in mass gathering health preparedness planning. These Collaborating Centres are working with WHO Regional and Country Offices to promote public health planning and positive legacy development as a key area of investment for mass gathering organizers. The Ministry of Health of
Saudi Arabia, Public Health England in the United Kingdom, the Institute of Public Health of Vojvodina in Serbia and the Disaster Research Centre and Flinders University in Australia are currently Collaborating Centres.

WHO is also working closely with international sporting federations, such as the International Olympic Committee, to encourage knowledge transfer between successive hosts and to make health preparedness a central pillar of any event.

Health planning, however, is only one component of the complex choreography of preparedness that needs to be undertaken. WHO's efforts to support health authorities in mass gathering health planning is in line with the all-hazard and whole-of-society approaches driving public health risk management under the International Health Regulations(2005). Mass gatherings, which are most often bound by immutable time frames, known years in advance, are test-beds for achieving better integration between sectors that can be applied in other public health contexts and emergencies. Furthermore, WHO has developed a framework for legacy research based on four areas of health system improvement:

- improved medical and hospital services

- $\quad$ strengthened public health system

- an enhanced living environment

- increased health awareness.

WHO has worked with a number of countries in the EMR including with the

'Technical Officer, Global Alert and Response Operations, Global Capacities, Alert and Response, World Health Organization, Geneva, Switzerland.

${ }^{2}$ Director, Global Capacities, Alert and Response, World Health Organization, Geneva, Switzerland. 
Ministry of Health of Iraq with which a workshop on mass gathering health preparedness was held in Amman in October 2012. An international team of experts from WHO and a representative from Public Health England worked with responsible health authorities from the Ministry of Health and District Health authorities from Karbala to improve health preparedness in the areas of on surveillance, prehospital casualty management, food and water safety, risk communication, coordination, preparedness and social mobilization. Likewise, WHO is currently working closely with Ministry of Health of the Kingdom of Saudi Arabia to manage risks associated with the emergence of the Middle East Respiratory Syndrome Coronavirus in the context of Hajj.

From the articles in this special edition on mass gathering, the reader will understand the complexity involved in planning a mass gathering. This collection of papers will provide a sense of the research being undertaken in the EMR to better understand the risks associated with mass gatherings and potential solutions to prevent and mitigate these risks during the event and achieve a long-term benefit from the experience. WHO will continue to work closely with any Member State on mass gathering health preparedness and planning.

\section{Information sources}

1. Resolutions and Decisions, Annexes. Executive Board, 130th Session, Geneva, 16-23 January 2012 (http://apps.who.int/gb/ ebwha/pdf_files/EB130-REC1/B130_REC1-en.pdf\#page=17, accessed 7 October 2013).

2. Global Alert and Response (GAR).Communicable disease alert and response for mass gatherings [webpage](http://www. who.int/csr/mass_gatherings/en/, accessed 7 October 2013).
3. Global Alert and Response (GAR).Communicable disease alert and response for mass gatherings. Key considerations [webpage] (http://www.who.int/csr/mass_gathering/en/index. html, accessed 7 October 2013)

4. International Health Regulations [webpage] (http://www. who.int/topics/international_health_regulations/en/, accessed 7 October 2013). 